\title{
Hypercoagulability and haemodynamic abnormalities in atrial fibrillation
}

Non-valvar atrial fibrillation confers a substantial risk for stroke and thromboembolism which is estimated to be between $4.5 \%$ and $12 \%$ per year depending on associated risk factors. ${ }^{12}$ Recent studies have established the value of warfarin as thromboprophylaxis in atrial fibrillation; however, this treatment carries with it the inconvenience of regular monitoring of anticoagulation intensity and the risk of bleeding. ${ }^{12}$ Attention has therefore focused on the development of alternative, safe, and convenient antithrombotic regimens, and appropriate thromboembolic risk stratification. For example, a low intensity fixed dose warfarin-aspirin regimen has certain attractions for convenience, but the recent third Stroke Prevention in Atrial Fibrillation study demonstrated the lower efficacy of this regimen compared with conventional, adjusted dose, warfarin therapy. ${ }^{3}$ Careful risk stratification has also been advocated to ensure that patients with atrial fibrillation at high risk of stroke and thromboembolism receive warfarin, while low risk patients are at least treated with aspirin. A strategy of prescribing warfarin to every patient with atrial fibrillation, irrespective of risk stratification, may increase risk of bleeding, particularly intracranial haemorrhage, and cause unnecessary inconvenience to patients. ${ }^{4}$ Most risk stratification can be performed on clinical criteria alone, with some refinement of risk stratification using echocardiography. ${ }^{2}$ However, other developments to assist thromboembolic risk stratification are needed; one area may be the study of various haemostatic and clotting indices that suggest a hypercoagulable state.

As long as 150 years ago, Virchow ${ }^{5}$ postulated three components for thrombogenicity: abnormalities in the blood vessel wall, blood flow, and blood constituents. These three basic postulates appear to be fulfilled in patients with atrial fibrillation. Clinical and echocardiographic criteria may help identify the first two of Virchow's basic postulates for thrombogenesis-abnormalities of vessels and blood flow, such as heart valve disease and cardiac impairment. It is also well recognised that atrial fibrillation confers a hypercoagulable state, satisfying the third of Virchow's postulates. ${ }^{6}$ The hypercoagulable state in chronic atrial fibrillation appears to be independent of the underlying aetiology or any structural heart disease, including left atrial size or left ventricular function..$^{7-9}$ Patients with paroxysmal atrial fibrillation have also been shown to demonstrate abnormalities of haemostasis ${ }^{1011}$ related to the duration of a paroxysm of atrial fibrillation and whether the patient was in atrial fibrillation at the time of sampling. ${ }^{11}$ Patients who are started on anticoagulation ${ }^{712}$ or cardioverted from atrial fibrillation to sinus rhythm ${ }^{13}{ }^{14}$ demonstrate significant changes in markers of haemostasis, suggesting a reduction in the hypercoagulable state.

The phenomenon of spontaneous echo contrast has been shown during paroxysms of atrial fibrillation, indicating atrial stasis. ${ }^{15}$ The relation of various haemostatic markers and the risk of stroke or thromboembolism to the presence of spontaneous echo contrast is further evidence for a correlation between a hypercoagulable state and intra-atrial stasis. ${ }^{16-20}$ Blood stasis within the left atria is also detected by atrial appendage dysfunction ${ }^{21} 22$ and this appears to be an additional contributory factor to thrombus formation.

In this issue of Heart, Heppell et $a l^{23}$ further evaluate the relation between haemostatic and haemodynamic parameters to the presence of left atrial thrombus in nonrheumatic atrial fibrillation. They initially found abnormalities in haemostatic markers in patients with left atrial thrombus compared with those without thrombus, consistent with the association between thrombogenesis and these markers. Furthermore, these abnormalities were significantly associated with haemodynamic abnormalities, as indicated by peak left atrial appendage velocity (but not peak mid-left atrial or mitral valve outflow velocity), and the presence of spontaneous echo contrast on transoesophageal echocardiography, suggesting intra-atrial blood stasis. The close association between haemostatic and haemodynamic abnormalities in atrial fibrillation are again consistent with Virchow's triad.

However, transoesophageal echocardiography is not $100 \%$ sensitive for the detection of intracardiac thrombi, and the presence of haemostatic abnormalities associated with likely haemodynamic appearances of stasis (spontaneous echo contract, reduced left atrial appendage velocity, etc), further strengthens the case that risk stratification of stroke or thromboembolism could be further refined using indices of hypercoagulability. The value of haemostatic markers as risk stratification in patients with atrial fibrillation requires further study. We need to know-for example, whether an abnormal marker of a hypercoagulable state, such as high fibrin D-dimer, will predict stroke, thromboembolism or death in patients with atrial fibrillation. ${ }^{6}$ Lack of prognostic data in patients with chronic atrial fibrillation raises the question of whether these abnormalities of haemostasis in patients with atrial fibrillation are a "cause or effect" phenomenon.

Prognostic value of some haemostatic markers is provided by evidence that some markers are predictive of mortality, progression of arterial disease, and cardiovascular events, from studies in normal healthy men and patients with peripheral vascular disease. For example, high plasma fibrin D-dimer (but within the "normal" range) predicts both arterial thrombotic events ${ }^{24-26}$ and postoperative thrombosis. ${ }^{27}$ Other haemostatic markers, such as plasma fibrinogen and von Willebrand factor also have prognostic implications in patients with ischaemic heart disease and hypertension, being associated with pro- 
gression or severity of disease and cardiac events. ${ }^{28} 29$ These abnormalities in haemostatic markers are therefore suggestive of a continuum that exists between health, "statistically" increased haemostatic abnormalities such as a prethrombotic or hypercoagulable state, and "overtly" increased clotting in acute thrombosis (or sometimes in acute extravascular fibrin formation, as follows injury or surgery) ${ }^{6}$

Nevertheless, there have been concerns whether peripheral measurements of various haemostatic markers are equivalent to levels of similar markers within the left atrium. ${ }^{30}{ }^{31}$ Indeed, a recent report suggests that increased regional left atrial coagulation activity-for example, in mitral stenosis, occurs in the presence of left atrial spontaneous echo contrast in either sinus rhythm or atrial fibrillation, and is associated with normal systemic coagulation activity. ${ }^{30}$ Nevertheless, some of these studies have been performed in relatively small numbers of patients, some of whom were in sinus rhythm or atrial fibrillation, and had treatments with various antithrombotic regimens (including aspirin, warfarin, and heparin), and the precise relation between left atrial and systemic coagulation activities are still unresolved. In cardiovascular disease, the process of thrombogenesis is also unlikely to be confined to the left atrium.

Where do we go from here? The evidence is fairly clear for the presence of a hypercoagulable state in atrial fibrillation $^{6}$ and increasing evidence such as that by Heppell et $a l^{3}$ point towards a relation between intra-atrial stasis and the haemodynamic abnormalities associated with atrial fibrillation. Ideally, we should ascertain whether various markers of haemostasis are predictive of stroke and thromboembolism in patients with atrial fibrillation, which would therefore complement clinical and echocardiographic risk stratification for thromboembolism. However, the value of haemostatic markers in assessing prognosis in many cardiovascular disorders (including atrial fibrillation) requires further, larger studies. Based on recent clinical trials, most patients with chronic atrial fibrillation should now be considered for prophylactic oral anticoagulation therapy, and so it may no longer be ethical to undertake a prospective study of the predictive nature of haemostatic abnormalities in patients with atrial fibrillation without any oral anticoagulant therapy. Perhaps such studies could be performed in patients with atrial fibrillation whose risks of thrombosis or bleeding do not justify anticoagulant prophylaxis. The relation of haemostatic markers to existing risk stratification could be further clarified-for example, by relating levels of these markers to other clinical and echocardiographic risk factors in large populations of patients with atrial fibrillation. These indices of hypercoagulability may be useful in the evaluation of new antithrombotic treatment regimens, for example, aspirin-warfarin combinations, ${ }^{2}$ low dose warfarin ${ }^{32}$ ticlopidine or clopidogrel, for patients with atrial fibrillation. The value of these markers in assisting the monitoring of anticoagulation intensity needs to be fully established.

Haemostasis Thrombosis and Vascular Biology Unit,

University Department of Medicine, City Hospital,

Birmingham B18 $7 Q H$, United Kingdom

e-mail: greg@cht.medicine.demon.co.uk

1 Atrial Fibrillation Investigators. Risk factors for stroke and efficacy of antithrombotic therapy in atrial fibrillation. Analysis of pooled data from five randomized controlled trials. Arch Intern Med 1994;154:1449-57. 2 Lip GYH, Lowe GDO. Antithrombotic therapy for atrial fibrillation. BMF

3 Stroke Prevention in Atrial Fibrillation Investigators. Adjusted dose war- farin versus low-intensity, fixed dose warfarin plus aspirin for high risk patients with atrial fibrillation: Stroke Prevention in Atrial Fibrillation III randomised clinical trial. Lancet 1996;348:633-8.

4 Stroke Prevention in Atrial Fibrillation Investigators. Warfarin versus aspirin for prevention of thromboembolism in atrial fibrillation: Stroke Prevention in Atrial Fibrillation II study. Lancet 1994;343:687-91.

5 Virchow R. Gesammelte abhandlungen zur wissenschaftichen medtzin. Frankfurt: Medinger Sohn \& Co, 1856:219-732.

6 Lip GYH. Does atrial fibrillation confer a hypercoagulable state? Lancet 1995;346:1313-14

7 Lip GYH, Lowe GDO, Rumley A, Dunn FG. Increased markers of thrombogenesis in chronic atrial fibrillation: effects of warfarin therapy. $B$ Heart f 1995; 73:527-33

8 Kumagai K, Fukunami M, Kitabatake A, Kamada T, Hoki N. Increased cardiovascular clotting in patients with chronic atrial fibrillation. $\mathcal{f} A m$
Coll Cardiol 1990;16:377-80.

9 Mitusch R, Siemens HJ, Garbe M, Wagner T, Sheikhzadeh A, Diederich $\mathrm{KW}$. Detection of a hypercoagulable state in nonvalvular atrial fibrillation and the effect of anticoagulant therapy. Thromb Haemostat 1996;75: 219-23.

10 Lip GYH, Lowe GDO, Rumley A, Dunn FG. Plasma fibrinogen and fibrin D-dimer levels in paroxysmal atrial fibrillation: evidence for intermediat levels of intravascular thrombogenesis. Am Heart f 1996;131:724-30.

11 Sohara H, Amitani S, Kurose M, Miyahara K. Atrial fibrillation activate platelets and coagulation in a time-dependent manner: a study in patients with paroxysmal atrial fibrillation. $\mathcal{F}$ Am Coll Cardiol 1997;29:106-12.

12 Asakura H, Hifumi S, Jokaji H, Saito M, Kumabashiri I, Uotani C, et al. Prothrombotic fragment F1 +2 and thrombin-antithrombin III complex
are useful markers of the hypercoagulable state in atrial fibrillation. Blood Coag Fibrinolys 1992;3:469-73.

13 Lip GYH, Rumley A, Dunn FG, Lowe GDO. Plasma fibrinogen and fibrin D-dimer levels in atrial fibrillation: effect of cardioversion to sinus rhythm. Int $f$ Cardiol 1995;51:245-53.

14 Abe Y, Kim J, Fukunami M, Ohmori M, Shimonagata T, Kumagai K, et al. Evidence for the intravascular hyperclotting state induced by atrial fibal. Evidence for the intravascular hyperclotting state induced by atrial

15 Obarski TP, Salcedo EE, Castle LW, Stewart WJ. Spontaneous echo contrast in the left atrium during paroxysmal atrial fibrillation. Am Heart $\mathcal{F}$ 1990;120:988-90.

16 Daniel WG, Nellesen U, Schroder E, Nonnast-Daniel B, Bednarski P, Nikutta $\mathrm{P}$, et al. Left atrial spontaneous echo contrast in mitral valve disease: an indicator for an increased thromboembolic risk. $\mathcal{F} \mathrm{Am}$ Coll Cardiol 1988;11:1204-11.

17 Black IW, Hopkins AP, Lee LCL, Walsh WF. Left atrial spontaneous echo contract: a clinical and echocardiographic analysis. $\mathcal{F}$ Am Coll Cardio 1991;18:398-404.

18 Black IW, Chesterman CN, Hopkins AP, Lee LCL, Chong BH, Walsh WF. Hematologic correlates of left atrial spontaneous echo contrast and thromboembolism in nonvalvular atrial fibrillation. $f \mathrm{Am}$ Coll Cardiol 1993;21:451-7.

19 Fatkin D, Herbert E, Feneley MP. Hematologic correlates of spontaneous echo contrast in patients with atrial fibrillation and implications for thromboembolic risk. Am F Cardiol 1994;73:672-6.

20 Tsai LM, Chen JH, Fang CJ, Lin LJ, Kwan CM. Clinical implications of left atrial spontaneous echo contrast in nonrheumatic atrial fibrillation. Am $\mathcal{F}$ Cardiol 1992;70:327-31.

21 Mugge A, Kuhn H, Nikutta P, Grote J, Lopez JAG, Daniel WG. Assessment of left atrial appendage function by biplane transoesophageal echocardiography in patients with nonrheumatic atrial fibrillation: identification of a subgroup of patients at increased embolic risk. $₹ \mathrm{Am} \mathrm{Coll}$ Cardiol 1994;23:599-607.

22 Mitusch R, Garbe M, Schmucker G, Schwabe K, Stierle U, Sheikhzadeh A. Relation of left atrial appendage function to the duration and A. Relation of left atriality of non-valvular atrial fibrillation. Am $f$ Cardiol 1995;75: reversibili.

23 Heppell RM, Berkin KE, McLenachan JM, Davies JA. Haemostatic and haemodynamic abnormalities associated with left atrial thrombosis in non-rheumatic atrial fibrillation. Heart 1997;77:407-11

24 Ridker PM, Hennekens CH, Cerskus A, Stampfer MJ. Plasma concentrations of cross-linked fibrin degradation product (D-dimer) and the risk of future myocardial infarction among apparently healthy men. Circulation 1994;90:2236-40.

25 Lowe GDO, Rumley A, Yarnell JWG, Sweetnam PM, Thomas HF. Fibrin D-dimer, von Willebrand factor and tissue plasminogen activator antigens are predictors of major ischaemic heart disease: the Caerphilly study. Blood Coag Fibrinolys 1995;6:156-7.

26 Fowkes FGR, Lowe GDO, Housley E, Rattray A, Rumley A, Elton RA, et al. Cross-linked fibrin degradation products, progression of peripheral arterial disease, and risk of coronary heart disease. Lancet 1993;342:84-6.

27 Rowbotham BJ, Whitaker AN, Harrison J, Murtaugh P, Reasbeck P, Bowie EJW. Measurement of cross-linked fibrin derivatives in patients undergoing abdominal surgery: use in the diagnosis of postoperative venous thrombosis. Blood Coag Fibrinolys 1992;3:25-31.

28 Jansson $\mathrm{JH}$, Nilsson TK, Johnson $\mathrm{O}$. von Willebrand factor in plasma: a novel risk factor for recurrent myocardial infarction and death. Br Heart $\mathcal{F}$ 1991;66:351-5.

29 Blann AD, Waite MI. von Willebrand factor and soluble E-selectin in hypertension: influence of treatment and value in prediction of progreshypertension: influence of treatment and value in predict
sion of atherosclerosis. Coronary Artery Dis 1996;7:143-7.

30 Peverill RE, Harper RW, Gelman J, Gan TE, Harris G, Smolich Determinants of increased regional left atrial coagulation activity in

31 Yamamoto K, Ikeda U, Seino Y, Mito H, Fujikawa H, Sekiguchi H, et al. Coagulation activity is increased in the left atrium of patients with mitral stenosis. F Am Coll Cardiol 1995;25:107-12.

32 Lip GYH, Lip PL, Zarifis J, Bareford D, Lowe GDO, Watson RDS, et al. Fibrin D-dimer and beta-thromboglobulin as markers of thrombogenesis and platelet activation in atrial fibrillation: effects of introducing ultralow dose warfarin and aspirin. Circulation 1996;94:425-31. 